\title{
ON THE SET OF VALUES OF A FINITE MEASURE
}

\author{
PAUL R. HALMOS
}

Let $X$ be any set, let $S$ be a $\sigma$-field of subsets of $X$ (that is, $S$ is a class of sets containing $X$ and closed under complementation and the formation of countable unions), and suppose that $\mu$ is a finite, non-negative, and countably additive measure defined for the sets of $\mathbf{S}$. Concerning such measures Kai Rander Buch has recently proved the following two statements. ${ }^{1}$

TheOREM 1. The set of values of $\mu$ is closed.

THEOREM 2. If $\mu$ and $\nu$ are two finite measures defined on the same $\sigma$-field $S$ of sets, then the set of all points of the form $(\mu(E), \nu(E))$, where $E \in S$, is a closed subset of the plane.

Buch's proofs are long and complicated and make use of an intricate construction involving the Cantor set in order to map the measure space $X$ on an interval. ${ }^{2}$ It is the purpose of this note to give direct and simple proofs of Theorems 1 and 2. It is worth remarking that (a) Theorem 1 is a trivial corollary of Theorem 2 (set $\nu(E)$ identically zero), (b) there does not seem to be a completely trivial proof of Theorem 2 from Theorem 1 based on elementary properties of product spaces, and (c) possibly both theorems can be made to appear as special cases of a theorem on measures whose values are suitably general entities (such as, say, elements of an ordered abelian group).

An atom of a measure space $X$ is a measurable set $E$ of positive measure such that for every measurable subset $F \subset E$ either $\mu(F)=0$ or $\mu(E-F)=0$. If $E$ is an atom of $X$ we may replace $X$ by the space whose points are the points of the complement of $E$ together with a single point of measure $\mu(E)$. Since the set of values of $\mu$ is not altered by this replacement we may and do assume that all atoms contain exactly one point. Since $\mu(X)<\infty, X$ can contain at most countably many distinct atoms. Let $Y$ be the union of the atoms of $X$ and write $Z=X-Y$.

Received by the editors August 9, 1946.

${ }^{1}$ Some investigations of the set of values of measures in abstract space, MatematiskFysiske Meddelelser vol. 21 (1945).

${ }^{2}$ Such constructions were previously employed for the same purpose by John von Neumann, Zur Operatorenmethode in der klassischen Mechanik, Ann. of Math. vol. 33 (1932) p. 602, and J. L. Doob, Stochastic processes with an integral-valued parameter, Trans. Amer. Math. Soc. vol. 44 (1938) p. 91. 
LEMMA 1. Every measurable set $E \subset Z$ of positive measure contains measurable subsets of arbitrarily small positive measure.

Proof. Since $E$ is not an atom there exists a measurable subset $F \subset E$ for which $0<\mu(F)<\mu(E)$. Write $E_{1}$ for that one of the two sets $F$ and $E-F$ whose measure is not greater than $\mu(E) / 2$. Similarly we may construct a set $E_{2} \subset E_{1}$ such that $0<\mu\left(E_{2}\right) \leqq \mu\left(E_{1}\right) / 2$, and proceed so on by induction.

Lемма 2. The set of values of $\mu$ on measurable subsets of $Z$ is the closed interval $0 \leqq x \leqq \mu(Z)$.

Proof. If $\mu(Z)=0$ there is nothing to prove. If $0<\alpha<\mu(Z)$, we may apply Lemma 1 to find a measurable set $E_{1} \subset Z$ such that $0<\mu\left(E_{1}\right) \leqq \alpha$. If the equality holds we are finished; if not we may apply Lemma 1 to find a measurable set $E_{2} \subset Z-E_{1}$ such that $0<\mu\left(E_{2}\right) \leqq \alpha-\mu\left(E_{1}\right)$. Proceeding in this way, by transfinite induction if necessary, we obtain a countable sequence of pairwise disjoint measurable sets the union of which has measure $\alpha .^{3}$

Lemma 3. The set of values of $\mu$ on measurable subsets of $Y$ is closed.

Proof. Let $y_{1}, y_{2}, \cdots$ be the points of $Y$. Let $\Gamma$ be the set of all sequences $\gamma=\left\{\epsilon_{1}, \epsilon_{2}, \cdots\right\}$ where $\epsilon_{i}=0$ or 1 . In the customary topology of Cartesian product spaces $\Gamma$ is a compact topological space and each of the functions $\epsilon_{i}=\epsilon_{i}(\gamma)$ is a continuous function. ${ }^{4}$ It follows from the finiteness of $\mu(Y)$ and the Weierstrass M-test that the function $\phi(\gamma)$ defined by the series

$$
\phi(\gamma)=\sum_{i=1}^{\infty} \epsilon_{i} \mu\left(y_{i}\right)
$$

is also a continuous function on $\Gamma$. Since a continuous image of a compact space is compact and therefore closed ${ }^{5}$ and since the image $\phi(\Gamma)$ is exactly the set of all values of $\mu$ on subsets of $Y$, the proof of Lemma 3 is complete.

It is not difficult to put together Lemmas 2 and 3 in order to prove Theorem 1. It is however a little more convenient not to do that directly but, with the proof of Theorem 2 in mind, to establish first two easy but mildly interesting topological lemmas.

\footnotetext{
${ }^{3}$ The device used in the proof of Lemma 2 finds frequent application in measure theory; it is called the method of exhaustion.

${ }^{4}$ See Solomon Lefschetz, Algebraic topology, Amer. Math. Soc. Colloquium Publications, vol. 27, New York, 1942, p. 19.

${ }^{5}$ See Lefschetz, op. cit. p. 18.
} 
LEMMA 4. Let $S$ be an arbitrary set and let $f$ be a function defined on $S$ and taking values in a topological space $R$. $A$ necessary and sufficient condition that there exist in $S$ a topology with respect to which $S$ is compact and $f$ is continuous is that the image $f(S)$ be a compact subset of $R$.

Proof. The necessity of the condition asserts merely that a continuous image of a compact space is compact. To prove sufficiency suppose that $f(S)$ is compact, and consider all those subsets of $S$ which are of the form $f^{-1}(U)$ where $U$ is an open set in $R$. Defining each such set to be open in $S$ makes $S$ into a topological space (without any separation axioms in general) on which $f$ is continuous. Since any open covering of $S$ is obviously induced by an open covering of $f(S)$, $S$ is compact with respect to the topology described.

LemMa 5. Suppose that $S$ is a set which is a compact space with respect to each of two topologies $T_{1}$ and $T_{2}$. Let $T$ be the weakest topology on $S$ (that is, the one with fewest open sets) whose open sets include all open sets of both $T_{1}$ and $T_{2}$. Then $S$ is compact with respect to $T$.

Proof. The class of all sets of the form $U V$, where $U$ is open with respect to $T_{1}$ and $V$ is open with respect to $T_{2}$, is a base of the open sets of $T$. If $S$ is covered by sets of this form then (because of the compactness hypotheses) it is covered by a finite number of the $U$ 's that occur and also by a finite number of the $V$ 's that occur. It follows therefore that $S$ is covered by the finite class of sets obtained by intersecting each one of the finite number of $U$ 's with each one of the finite number of $V$ 's.

Lemma 6. If $\nu_{1}$ and $\nu_{2}$ are two measures defined on the same $\sigma$-field $\mathbf{S}$ of sets and if the set of values of each of them is a closed and bounded set on the line, then the set of all points of the form $\left(\nu_{1}(E), \nu_{2}(E)\right)$, where $E \in S$, is a closed and bounded subset of the plane.

Proof. By Lemma 4 we may introduce a topology $T_{i}$ into the space $S$ of measurable sets so that $S$ is compact and $\nu_{i}$ is continuous, $i=1,2$. By Lemma $6, S$ is compact with respect to the weakest topology $T$ which is stronger than both $T_{1}$ and $T_{2}$, and it is clear that the introduction of additional open sets does not affect the continuity of $\nu_{1}$ and $\nu_{2}$. It follows that the point $\left(\nu_{1}(E), \nu_{2}(E)\right)$ depends continuously on $E$ (with respect to the compact topology $T$ ) and that consequently the set of all such points is compact. This completes the proof of Lemma 6.

We return now to the notation of Lemmas 2 and 3. If for every $E \in S$ we write $\nu_{1}(E)=\mu(E Y)$ and $\nu_{2}(E)=\mu(E Z)$ then Lemmas 2 and 3 
assert that the conditions of Lemma 6 are satisfied by $\nu_{1}$ and $\nu_{2}$. It follows that the set of all points of the form $(\mu(E Y), \mu(E Z))$ is a compact set. Since the function $f(s, t)=s+t$ is continuous, it follows that the set of all numbers of the form

$$
\mu(E)=\mu(E Y)+\mu(E Z)
$$

is also compact, and this proves Theorem 1 . Theorem 2 is an immediate corollary of Theorem 1 and Lemma 6 . The method of proof shows, incidentally, that the obvious generalization of Theorem 2 from two to $n$ dimensions is also true.

University of Chicago 